\title{
Spontaneous Unilateral Tubal Twin Ectopic Pregnancy
}

\author{
Hannah E. Lategan, MBBS, ${ }^{1}$ Veronica C. Gillispie, MD, FACOG ${ }^{1,2}$ \\ ${ }^{1}$ The University of Queensland Faculty of Medicine, Ochsner Clinical School, New Orleans, LA ${ }^{2}$ Department of Obstetrics and Gynecology, \\ Ochsner Clinic Foundation, New Orleans, LA
}

Background: Unilateral tubal twin pregnancies occur in approximately 1 of every 125,000 spontaneous pregnancies. Because of the paucity of data, little guidance for the appropriate management of a twin tubal ectopic pregnancy is available.

Case Report: A 40-year-old female presented to the emergency department (ED) with a 2-day history of vaginal bleeding associated with lower abdominal pain. The patient was hemodynamically stable with signs of an acute abdomen. Pelvic examination elicited clinical findings consistent with potential ectopic pregnancy. Urine pregnancy test in the ED was positive. Serum betahuman chorionic gonadotropin was $23,359 \mathrm{mIU} / \mathrm{mL}$, and transvaginal ultrasound showed findings concerning for a twin ectopic pregnancy. Diagnostic laparoscopy with right salpingostomy was successful, and the patient had an uncomplicated postoperative course.

Conclusion: Healthcare providers should have a high index of clinical suspicion for ectopic pregnancies. Unilateral tubal twin ectopic pregnancies, while rare, can be adequately diagnosed with transvaginal ultrasound. Laparoscopic salpingostomy provides conservative and successful treatment of unilateral tubal twin gestation with short recovery time. Medical treatment with methotrexate has not been adequately studied in this clinical scenario, but further exploration of this management modality should be considered.

Keywords: Pregnancy-ectopic, pregnancy-tubal, twin ectopic

Address correspondence to Veronica C. Gillispie, MD, FACOG, Department of Obstetrics and Gynecology, Ochsner Clinic Foundation, Ochsner Baptist Medical Center, 4429 Clara Street, Suite 500, New Orleans, LA 70115. Tel: (504) 842-9617. Email: vgillispie@ochsner.org

\section{INTRODUCTION}

Ectopic pregnancy accounts for approximately 1\%-2\% of first-trimester pregnancies and may be the cause of up to $6 \%$ of pregnancy-related deaths. ${ }^{1}$ Risk factors for an ectopic pregnancy include prior tubal surgery, pelvic inflammatory disorder, peritubal adhesions (following appendicitis or endometriosis), congenital tubal anomalies (ie, in utero diethylstilbestrol exposure), artificial reproductive therapy, and smoking. Although contraceptive methods overall decrease the risk of ectopic pregnancy by preventing all pregnancies, intrauterine devices (IUDs) may actually increase the risk. ${ }^{1}$ Early detection of ectopic pregnancy has improved via urine/serum beta-human chorionic gonadotropin (bHCG) testing and transvaginal ultrasound, thus decreasing associated morbidity and mortality. Tubal ectopic pregnancies comprise $95 \%$ of ectopic pregnancies. Within this incidence lies the even rarer occurrence of tubal twin ectopic pregnancy, with incidence rates estimated to be 1 in $725-1,580$ of tubal pregnancies. ${ }^{2}$ Unilateral tubal twin pregnancies occur in approximately 1 of every 125,000 spontaneous pregnancies. ${ }^{3}$ A diagnosis of prior ectopic pregnancy increases the risk of subsequent ectopic pregnancy by $10 \% .^{1}$

Management options for ectopic pregnancy are medical management via methotrexate or surgical management.
Because of the paucity of data, little guidance for the appropriate management of a twin tubal ectopic pregnancy is available.

\section{CASE REPORT}

A 40-year-old female gravida 1 para 1001 presented to the emergency department (ED) with a 2-day history of vaginal bleeding associated with lower abdominal pain. The patient stated that she had filled several pads with dark red blood and large clots that looked like "tissue." She denied any vaginal pain or discharge, dysuria, and frequency or urgency of urination. The patient also denied constitutional symptoms, as well as gastrointestinal symptoms. Her last menstrual period was 6 weeks prior to presentation. She denied any history of sexually transmitted disease. She had no significant medical history, and her surgical history was only significant for cesarean section for her first child (17 years previously). The patient denied taking any medications and is a nonsmoker.

On physical examination, the patient was afebrile with stable vital signs. On abdominal examination, the patient was nontender, soft, and nondistended with no signs of rebound tenderness or guarding. Examination of the external genitalia showed no abnormalities. Pelvic examination revealed some dark brown blood in the vaginal vault with no active bleeding. 

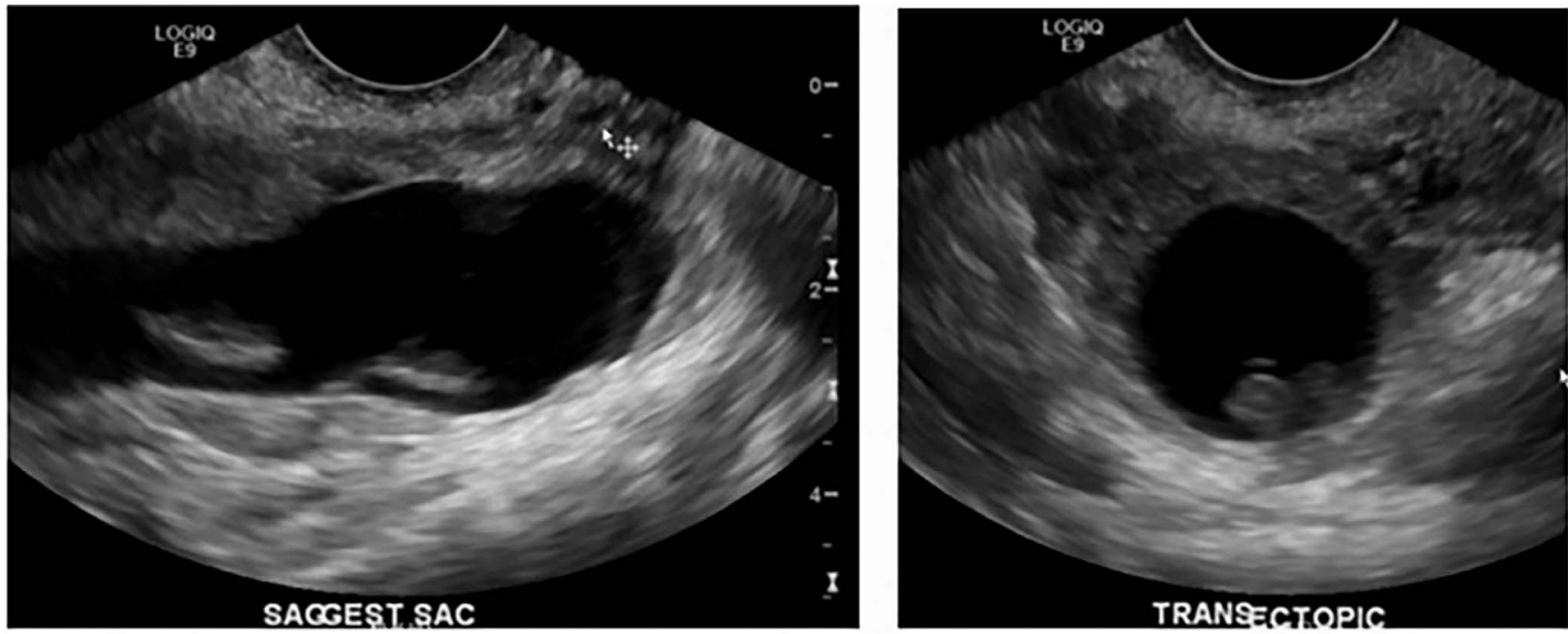
A

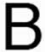

Figure. A: Transvaginal ultrasound sagittal view shows the right fallopian tube containing 2 discrete gestational sacs. B: Transvaginal ultrasound transverse view shows the right fallopian tube containing 2 discrete gestational sacs.

The patient had no cervical motion tenderness. Bimanual examination revealed a uterus of 6 -week size with a closed cervical os. No adnexal masses were present; however, the patient acknowledged tenderness on palpation of the right adnexa.

In the ED, a urine pregnancy test was positive. Blood work showed serum bHCG of 23,359 $\mathrm{mlU} / \mathrm{mL}$, hemoglobin of $10.4 \mathrm{~g} / \mathrm{dL}$, hematocrit of $32.4 \%$, and $\mathrm{O}$ positive blood type. Transvaginal ultrasound (Figure) revealed a uterus measuring $8.3 \mathrm{~cm}$ in length and $3.9 \times 4.5 \mathrm{~cm}$ in transverse dimensions with no intrauterine gestational sac; however, 2 closely opposed gestational sacs, each containing a fetal pole, were seen in the right adnexal region separate from the ovary. The left ovary was not identified, and no left adnexal abnormalities were seen. Fetal heart tones were not detected at either fetal pole. No significant amount of free fluid was present within the pelvis. Overall, these findings indicated a right tubal ectopic twin pregnancy of average ages of 8 weeks and 2 days and 7 weeks and 6 days, respectively.

The patient was emergently taken to the operating room for diagnostic laparoscopy with right salpingostomy. Intraoperative findings were consistent with the preoperative diagnosis. The patient's postoperative course was uncomplicated, and she was discharged on postoperative day zero. Histopathology confirmed the diagnosis. Serial bHCG levels were checked until the level was $<5 \mathrm{mIU} / \mathrm{mL}$.

\section{DISCUSSION}

From the report in 1891 by De Ott ${ }^{4}$ through 1990, only 98 cases of unilateral tubal twin pregnancy had been described in the literature, most of which were diagnosed intraoperatively or postoperatively. ${ }^{5}$ The 101 st case of unilateral twin ectopic pregnancy was described only in $2001 .{ }^{6}$ In 2006, Rolle et al reported that only 8 cases of unilateral tubal twin pregnancy in the literature had been diag- nosed preoperatively. ${ }^{7}$ In the current case, the treatment team was able to establish with reasonable certainty that the patient had a unilateral twin ectopic pregnancy prior to surgery.

The literature includes case reports describing twin ectopic gestations following salpingectomies and other tubal surgeries, as well as others following a history of pelvic infection or artificial reproductive therapy. This patient, however, had no history of tubal surgery, pelvic infection, artificial insemination, tubal anomalies, IUDs, or smoking. In all women of reproductive age, even those with no known risk factors, physicians should operate with high clinical suspicion of potential ectopic pregnancy.

Because of the low incidence of spontaneous unilateral tubal twin pregnancies, no consensus has been reached as to the best option for management. In cases similar to ours, laparotomy and laparoscopic salpingectomy have been successfully performed. ${ }^{8}$ Only one other case report describes management via laparoscopic salpingostomy as achieved in our case. Only 2 cases of medical management with methotrexate have been documented in hemodynamically stable patients with unruptured unilateral tubal twin pregnancies, and both were successful. 9,10 $^{9}$

\section{CONCLUSION}

Even in the absence of the accepted risk factors, the possibility of unilateral twin ectopic pregnancy should be considered. Retrospectively in this case, treatment with methotrexate may have been an option; however, with extremely limited data on the use of medical management, methotrexate may not have been appropriate, but this question is an imperative for future study.

\section{ACKNOWLEDGMENTS}

The authors have no financial or proprietary interest in the subject matter of this article. 


\section{REFERENCES}

1. Cunningham FG, Leveno KJ, Bloom SL, et al. Ectopic pregnancy. In: Cunningham FG, Leveno KJ, Bloom SL, et al, eds. Williams Obstetrics. 24th ed. New York, NY: McGraw-Hill Education; 2014:377-395.

2. Svirsky R, Maymon R, Vaknin Z, et al. Twin tubal pregnancy: a rising complication? Fertil Steril. 2010 Oct;94(5):

1910.e13-1910.e16. doi: 10.1016/j.fertnstert.2010.03.017.

3. Dede M, Gezginç K, Yenen M, et al. Unilateral tubal ectopic twin pregnancy. Taiwan J Obstet Gynecol. 2008 Jun;47(2):226-228. doi: 10.1016/S1028-4559(08)60089-3.

4. De Ott D. A case of unilateral tubal twin gestation. Ann Gynécol Obstét. 1891;36:304.

5. Sherer DM, Liberto L, Woods JR Jr. Preoperative sonographic diagnosis of a unilateral tubal twin gestation with documented fetal heart activity. J Ultrasound Med. 1990 Dec;9(12):729-731.
6. Göker EN, Tavmergen E, Ozçakir HT, Levi R, Adakan S. Unilateral ectopic twin pregnancy following an IVF cycle. $J$ Obstet Gynaecol Res. 2001 Aug;27(4):213-215.

7. Rolle CJ, Wai CY, Bawdon R, Santos-Ramos R, Hoffman B. Unilateral twin ectopic pregnancy in a patient with a history of multiple sexually transmitted infections. Infect Dis Obstet Gynecol. 2006;2006:10306.

8. Sur SD, Reddy K. Spontaneous unilateral tubal twin pregnancy. J R Soc Med. 2005 Jun;98(6):276.

9. Karadeniz RS, Dilbaz S, Ozkan SD. Unilateral twin tubal pregnancy successfully treated with methotrexate. Int $J$ Gynaecol Obstet. 2008 Aug;102(2):171. doi: 10.1016/j.ijgo.2008.03.012.

10. Arikan DC, Kiran G, Coskun A, Kostu B. Unilateral tubal twin ectopic pregnancy treated with single-dose methotrexate. Arch Gynecol Obstet. 2011 Feb;283(2):397-399. doi: 10.1007/s00404-010-1449-6.

This article meets the Accreditation Council for Graduate Medical Education and the American Board of Medical Specialties Maintenance of Certification competencies for Patient Care and Medical Knowledge. 